\title{
A SCHWARZ LEMMA FOR BOUNDED SYMMETRIC DOMAINS
}

ADAM KORÁNYI ${ }^{1}$

The purpose of this note is to generalize Pick's invariant formulation of the classical Schwarz lemma. For the four classical types of bounded symmetric domains such a generalization was given by K. H. Look [3]; the present treatment will be independent of classification theory and will also include the exceptional Cartan domains. The results are also independent of the particular realization of the domain in $C^{n}$, they depend only on its structure as a hermitian manifold. The results will therefore be formulated for hermitian symmetric spaces of noncompact type; these are known to be in one-to-one correspondence with the holomorphic equivalence classes of bounded symmetric domains. We shall make use of Harish-Chandra's canonical realization of the hermitian symmetric spaces as bounded domains; this could perhaps be avoided, but it makes the proofs considerably simpler.

In the following $M=G / K$ will be a hermitian symmetric space of noncompact type; the identity coset will be denoted by $p_{0}, \mathfrak{g}$ and $\mathfrak{l}$ will denote the Lie algebras of $G$ and $K$, respectively, and $H_{\alpha}$, $E_{\alpha}, \cdots$ will be a Weyl basis of $g$ with respect to a Cartan subalgebra of $\mathfrak{g}$ contained in $\mathfrak{f}$. By a result of Harish-Chandra there exists a set $\Delta$ of strongly orthogonal roots of $\mathfrak{g}$ such that $\mathfrak{a}=\sum_{\alpha \in \Delta} R\left(E_{\alpha}+E_{-\alpha}\right)$ is a Cartan subalgebra of the symmetric pair $(\mathfrak{g}, \mathfrak{f})$. So every point $p \in M$ can be represented in the form $p=k \exp \left(\sum_{\alpha \in \Delta} t_{\alpha}\left(E_{\alpha}+E_{-\alpha}\right)\right) \cdot p_{0}$ with $k \in K, t_{\alpha} \geqq 0$.

For any $p, q \in M$ we denote by $d(p, q)$ the distance of $p$ and $q$ in the metric induced by the hermitian structure of $M$. In any realization of $M$ as a complex domain this is the Bergman metric. We denote by $d^{*}(p, q)$ the Carathéodory distance, which is defined by

$$
d^{*}(p, q)=\sup _{f \in F} d_{U}(f(p), f(q)),
$$

where $F$ is the family of all holomorphic maps of $M$ into the unit $\operatorname{disc} U \subset C$, and $d_{U}$ is the Poincaré-Bergman distance function on $U$.

Lemma. Let $p=\exp \left(\sum_{\alpha \in \Delta} t_{\alpha}\left(E_{\alpha}+E_{-\alpha}\right)\right) \cdot p_{0}, t_{\alpha} \geqq 0(\alpha \in \Delta)$. Then

Received by the editors February 15, 1965.

${ }^{1}$ Supported in part by contract DA 31124 ARO(D)-30. 


$$
\begin{aligned}
d\left(p_{0}, p\right) & =\left(\sum_{\alpha \in \Delta} t_{\alpha}^{2}\right)^{1 / 2}, \\
d^{*}\left(p_{0}, p\right) & =\operatorname{Max}_{\alpha \in \Delta} t_{\alpha} .
\end{aligned}
$$

Proof. The first statement follows from the known fact that the orbits of $p_{0}$ under one-parameter groups generated by elements of $a$ are geodesics; thus

$$
\exp \left(s\left(\sum_{\alpha \in \Delta} t_{\alpha}^{2}\right)^{-1 / 2} \sum_{\alpha \in \Delta} t_{\alpha}\left(E_{\alpha}+E_{-\alpha}\right)\right) \cdot p_{0}\left(0 \leqq s \leqq\left(\sum_{\alpha \in \Delta} t_{\alpha}^{2}\right)^{1 / 2}\right)
$$

is a geodesic segment in arc-length parameters connecting $p_{0}$ and $p$.

For the second statement we use the Harish-Chandra realization of $M$ as a bounded domain. We denote by $\Phi$ the set of positive roots of $\mathfrak{g}$ which are not roots of $\mathfrak{f}$, and by $\mathfrak{p}^{-}$the complex subspace of $\mathfrak{g}^{C}$ spanned by the vectors $E_{-\alpha}(\alpha \in \Phi)$. The Harish-Chandra realization $\eta: M \rightarrow p^{-}$is given for any $p=k \exp \left(\sum_{\alpha \in \Delta} t_{\alpha}\left(E_{\alpha}+E_{-\alpha}\right)\right) \cdot p_{0}$ by $\eta(p)$ $=\operatorname{ad}(k) \sum_{\alpha \in \Delta} r_{\alpha} E_{-\alpha}$, where $r_{\alpha}=\tanh t_{\alpha}(\alpha \in \Delta)$. We denote the domain $\eta(M) \subset p^{-}$by $D$.

Now let $p$ be as in the statement of the Lemma, and let $\alpha_{0} \in \Delta$ be such that $t_{\alpha_{0}}=\operatorname{Max}_{\alpha \in \Delta} t_{\alpha}$. We write $r_{\alpha_{0}}=\tanh t_{\alpha_{0}}$. Let $f: M \rightarrow U$ be a holomorphic function such that $f\left(p_{0}\right)=0$. Defining the function $\phi: U \rightarrow U$ by $\phi(z)=f\left(\eta^{-1}\left(z r_{\alpha_{0}}^{-1} \eta(p)\right)\right)$ we have, by the classical Schwarz lemma, $|\phi(z)| \leqq|z|$ for all $z \in U$. In particular, for $z=r_{\alpha_{0}}$, it follows that $|f(p)| \leqq r_{\alpha_{0}}$. In the definition of the Caratheodory distance it is sufficient to consider functions $f \in F$ such that $f\left(p_{0}\right)=0$, since $U$ is homogeneous. Hence, from what we just proved it follows that $d^{*}\left(p_{0}, p\right) \leqq d_{U}\left(0, r_{\alpha_{0}}\right)=t_{\alpha_{0}}$.

On the other hand, let $g: D \rightarrow U$ be defined by $g\left(\sum_{\alpha \in \Phi} z_{\alpha} E_{-\alpha}\right)=z_{\alpha_{0}}$, and let $f_{1}=g \circ \eta$. Then $f_{1} \in F$, and $d^{*}\left(p_{0}, p\right) \geqq d_{U}\left(f_{1}\left(p_{0}\right), f_{1}(p)\right)$ $=d_{U}\left(0, r_{\alpha_{0}}\right)=t_{\alpha_{0}}$, finishing the proof of the Lemma.

Proposition 1. Let $M$ be a hermitian symmetric space of rank $l$ and let $f: M \rightarrow M$ be a holomorphic function. Then, for any $p, q \in M$,

$$
d(f(p), f(q)) \leqq l^{1 / 2} d(p, q) .
$$

The constant $l^{1 / 2}$ is the best possible.

Proof. Given any pair of points $p_{1}, p_{2} \in M$ we can find an element $g$ in $G$ such that $g p_{1}=p_{0}, g p_{2}=\exp \left(\sum_{\alpha \in \Delta} t_{\alpha}\left(E_{\alpha}+E_{-\alpha}\right)\right) \cdot p_{0}$. By the Lemma it follows that

$$
d(f(p), f(q)) \leqq l^{1 / 2} d^{*}(f(p), f(q)) \leqq l^{1 / 2} d^{*}(p, q) \leqq l^{1 / 2} d(p, q)
$$


proving the first statement.

To see that $l^{1 / 2}$ is best possible, let $p=\exp t\left(E_{\alpha_{0}}+E_{-\alpha_{0}}\right) \cdot p_{0}$ with some $t>0$ and $\alpha_{0} \in \Delta$. Define $g: D \rightarrow D$ by $g\left(\sum_{\alpha \in \Phi} z_{\alpha} E_{-\alpha}\right)$ $=z_{\alpha_{0}} \sum_{\alpha \in \Delta} E_{-\alpha}$, and let $f=\eta^{-1} \circ g \circ \eta$. By Lemma 1 we have $d\left(p_{0}, p\right)$ $=t$ and $d\left(f\left(p_{0}\right), f(p)\right)=l^{1 / 2} t$, finishing the proof.

Remark. The Proposition remains true, by the same proof, for holomorphic functions $f: M_{1} \rightarrow M_{2}$ where $M_{1}, M_{2}$ are hermitian symmetric spaces and $l$ is the rank of $M_{2}$.

Next we give an infinitesimal formulation of Proposition 1; here we are also able to prove an analogue of the "strong form" of the classical Schwarz lemma (cf. [3]). For every $p \in M$ we denote by $M_{p}$ the space of real tangent vectors at $p . M_{p}$ is a complex Euclidean space under the hermitian structure of $M$, we denote the length of a vector $X \in M_{p}$ by $\|X\|$.

Proposition 2. Let $M$ be a hermitian symmetric space of rank $l$ and let $f: M \rightarrow M$ be a holomorphic function. Then for all $p \in M$ and $X \in M_{p}$ we have $\|d f(X)\| \leqq l^{1 / 2}\|X\|$, the constant $l^{1 / 2}$ being the best possible.

If there exists a point $p \in M$ such that $\|d f(X)\| \geqq\|X\|$ for all $X \in M_{p}$, then $f$ is a holomorphic automorphism of $M$.

Proof. The first statement follows from Proposition 1. To prove the second statement, let $g \in G$ be such that $g p=f(p)$. Then $h=g^{-1} \circ f$ maps $M$ onto itself and keeps $p$ fixed; since $g^{-1}$ is an isometry, the hypothesis implies $\|d h(X)\| \geqq\|X\|$ for all $X \in M_{p}$, and hence $\left|\operatorname{det}(d h)_{p}\right|$ $\geqq 1$. By a well-known theorem of $\mathrm{H}$. Cartan and Carathéodory (e.g. $[1$, Chapter 1]) it follows that $h$, and therefore also $f$, is a holomorphic automorphism of $M$, finishing the proof.

If $l=1$, we have the following sharper version of the "strong form."

Propositron 3. Let $M$ be a hermitian symmetric space of rank 1 and dimension $n$, and let $f: M \rightarrow M$ be a holomorphic function. If there exists $a$ point $p \in M$ and $n$ complex-linearly independent vectors $X_{1}, \cdots, X_{n}$ $\in M_{p}$ such that $\left\|d f\left(X_{i}\right)\right\|=\left\|X_{i}\right\|(i=1, \cdots, n)$, then $f$ is a holomorphic automorphism of $M$.

Proof. Let $g \in G$ be such that $g p=f(p)$, and let $h=g^{-1} \circ f$. By Proposition 2, $(d h)_{p}$ is a linear contraction of the complex Euclidean space $M_{p}$. Denoting the adjoint transformation by $(d h)_{p}^{*}$ it follows that $A=I-(d h)_{p}(d h)_{p}^{*}$ is a positive semidefinite linear transformation on $M_{p}$. By our hypothesis $\left\|A X_{i}\right\|=0(i=1, \cdots, n)$; this now implies $A=0$. It follows that $(d h)_{p}$ is unitary, whence $\left|\operatorname{det}(d h)_{p}\right|=1$, and by 
the above mentioned theorem of Cartan and Carathéodory the proof is finished.

\section{REFERENCES}

1. S. Bochner and W. T. Martin, Several complex variables, Princeton Univ. Press, Princeton, N. J., 1948.

2. S. Helgason, Differential geometry and symmetric spaces, Academic Press, New York, 1962.

3. K. H. Look, Schwarz lemma and analytic invariants, Sci. Sinica 7 (1958), 453504.

Princeton University

\section{A CHARACTERIZATION OF TAME 2-SPHERES IN $E^{3}$}

\section{A. PERSINGER ${ }^{1}$}

In this note, the tame 2-spheres in $E^{3}$ are characterized partly in terms of homology and the arcs they contain. In a similar way, the compact 2-manifolds with boundary are characterized. If $K$ is a finite topological 2-complex in $E^{3}$ and $v$ is a vertex of $K$, then St $v$ is the star of $v, \dot{\mathrm{S} t} v$ is the open $\operatorname{star}$ of $v$, and Lk $v=\mathrm{St} v-\dot{\mathrm{St}} v$ is the link of $v$. The trivial 1-dimensional homology group of $K$ will be denoted by $H_{1}(K)=0$.

An $n$-manifold with boundary is a separable metric space such that each point has a neighborhood whose closure is topologically equivalent to a closed $n$-cell.

THEOREM 1. Let $K$ be a finite topological 2-complex in $E^{8}$ such that

(i) $K$ is connected,

(ii) Lk $v$ is connected for each vertex $v$ in $K$,

(iii) $H_{1}(K)=0$, and

(iv) $K$ contains only tame arcs.

Then $K$ is either a disk or a 2-sphere.

Proof. Since $K$ contains no wild arcs and $L k v$ is connected, each 1 -simplex in $K$ lies on exactly one or two 2 -simplices in $K$ [2]. Since

Presented to the Society, March 29, 1965 under the title $A$ characterization of tame 2-spheres; received by the editors May 31, 1965.

1 These results form a part of the author's doctoral dissertation written at the Virginia Polytechnic Institute in 1964 under the direction of Professor P. H. Doyle. 